\title{
Study of the expression and function of ACY1 in patients with colorectal cancer
}

\author{
BING YU ${ }^{1,2}$, XUEZHONG LIU ${ }^{3}$, XIUZHEN CAO ${ }^{2}$, MINGYUE ZHANG ${ }^{2}$ and HONG CHANG ${ }^{1}$ \\ ${ }^{1}$ Department of Hepatobiliary Surgery, Shandong Provincial Hospital Affiliated to Shandong University, \\ Jinan, Shandong 250021; ${ }^{2}$ Department of Colorectal Surgery, Tai'an Central Hospital, Tai'an, Shandong 271000; \\ ${ }^{3}$ Department of Gastrointestinal Surgery, Liaocheng People's Hospital, Liaocheng, Shandong 252000, P.R. China
}

Received February 22, 2016; Accepted October 19, 2016

DOI: $10.3892 / 01.2017 .5702$

\begin{abstract}
Aminoacylase 1 (ACY1) is important for regulating the proliferation of numerous types of cancer. However, the expression and mechanisms underlying the function of ACY1 in colorectal cancer remain unclear. In order to investigate the expression and function of ACY1 in colorectal cancer, tumor tissue and blood samples were collected for analysis from 132 patients diagnosed with colorectal cancer. Reverse transcription-quantitative polymerase chain reaction analysis and western blotting identified significantly increased expression of ACY1 mRNA in colorectal tumor tissue $(\mathrm{P}<0.05$ vs. adjacent normal tissue) and notably increased ACY1 protein levels. This ACY1 mRNA expression was found to be positively correlated with tumor stage. In addition, plasma ACY1 concentration was increased in patients with colorectal cancer compared with healthy controls. Furthermore, in vitro knockdown of ACY1 in human colorectal cancer HT-29 cells was shown to inhibit proliferation and increase apoptosis. This effect was found to be associated with the activation of ERK1 and TGF- $\beta 1$ signaling. In conclusion, the results of the present study suggest that ACY1 promotes tumor progression, and thus may be a potential target for the diagnosis and treatment of colorectal cancer.
\end{abstract}

\section{Introduction}

Colorectal cancer, the third most common cancer worldwide, is a major public health issue in China and globally (1). Although advances have been made in surgical and medical treatments, $\sim 40 \%$ of patients with colorectal cancer succumb to the cancer (2). The early diagnosis and assessment of tumors is an

Correspondence to: Dr Hong Chang, Department of Hepatobiliary Surgery, Shandong Provincial Hospital Affiliated to Shandong University, 324 Jingwuweiqi Street, Jinan, Shandong 250021, P.R. China

E-mail: hongchang1102@163.com

Key words: aminoacylase 1, colorectal cancer, tumor proliferation, clinical diagnosis area of intense study; however, it is a complex issue. Previous studies indicate that tumor cells of different clinical stages may exhibit different expression levels of certain biomarkers, which could be used as a target for the diagnosis or treatment of the tumor (3-5). In addition, with ongoing and advancing research into tumor biology, new targets are being identified and studied, including aminoacylase 1 (ACY1) (6).

ACY1 is a cytosolic enzyme that deacylates the $\alpha$-acylated amino acid from the N-terminal peptide of intracellular proteins (6). Following this, terminal acetylated proteins are more stable (7-9). In addition to its function in scavenging $\mathrm{N}$-acylated amino acids, ACY1 has been studied in a number of types of human cancer. In small cell lung cancer (SCLC), renal cell carcinoma and liver cancer ACY1 expression has been demonstrated to be significantly reduced (10-13), suggesting that ACY1 serves a role in inhibiting tumorigenesis. Limited studies have investigated the detailed function and mechanism of ACY1 in tumor proliferation, and certain previous studies have reported that activation of ERK $1 / 2$ and expression of TGF- $\beta$ may be implicated in this process (13-16). However, the role of ACY1 in gastrointestinal cancer remains unclear. In the present study, the function and regulation of ACY1 in colorectal cancer was investigated through analyzing clinical samples.

\section{Materials and methods}

Patients and clinical specimens. In total, 160 patients who underwent colectomy in the Department of Gastrointestinal Surgery at Liaocheng People's Hospital (Liaocheng, China) between January 2010 and January 2015 were included in the present study and evaluated for the presence of tumor cells. This led to 28 cases being excluded for the absence of tumor cells. Tumor cells were found in the remaining 132 cases. Colorectal adenocarcinoma and adjacent normal colorectal tissue specimens were collected from these patients, along with serum samples, between January 2010 and January 2015. The tissues were immediately put into ice-cold RPMI-1640 medium (Hyclone; GE Healthcare Life Sciences, Logan, UT, USA) and transported to the lab for further protein and RNA extraction. The serum and tissue specimens were collected and stored at $-80^{\circ} \mathrm{C}$ for ELISA, western blotting and reverse transcription-quantitative polymerase chain reaction 
(RT-qPCR) analysis. In addition, 120 cases of serum samples from healthy volunteers were collected (between January 2010 and January 2015) as a control for the serum of the patients. Clinicopathological characteristics of the patients and control group are listed in Table I. This protocol was approved by the Institutional Review Board for Human Research of the School of Medicine, Shandong University (Jinan, China) and informed consent was obtained from all patients.

ACY1 RT-qPCR. Total RNA was isolated from the tissues using TRIzol reagent (Thermo Fisher Scientific, Inc., Waltham, MA, USA) and then reverse transcribed using the PrimeScript RT reagent kit (Clontech Laboratories, Inc., Mountain View, CA, United States), according to the manufacturer's protocol. Subsequently, the mRNA expression of ACY1 and $\beta$-actin (the internal control) was quantified through qPCR analysis (Applied Biosystems). The following primers, described previously $(13,14)$, were used: ACY1 forward, 5'-GGCTGC ATGAGGCTGTGTT-3' and reverse, 5'-CTTGGCACTGGT TGGGATG-3'; and $\beta$-actin forward, 5'-TGGCACCCAGCA CAATGAA-3' and reverse, 5'-CTAAGTCATAGTCCGC CTAGAAGCA-3'. The thermocycling conditions were as follows, for a total of 40 cycles: Denaturing at $95^{\circ} \mathrm{C}$ for $30 \mathrm{sec}$; annealing at $60^{\circ} \mathrm{C}$ for $5 \mathrm{sec}$; and extension at $72^{\circ} \mathrm{C}$ for $45 \mathrm{sec}$. The $2^{-\Delta \Delta \mathrm{Cq}}$ method was used for the quantification of the PCR results $(13,17,18)$.

Cell culture and small interfering RNA (siRNA) silencing. Human colorectal adenocarcinoma HT-29 cells were used for in vitro analysis of the function and regulation of ACY1. The cells were cultured in the McCoy's 5A Medium (cat. no. 16600; Gibco; Thermo Fisher Scientific, Inc.) supplemented with 10\% fetal bovine serum (FBS; Hyclone; GE Healthcare Life Sciences), $100 \mathrm{U} / \mathrm{ml}$ penicillin and $100 \mathrm{~g} / \mathrm{ml}$ streptomycin, as previously described (19). Cells were incubated at $37^{\circ} \mathrm{C}$ in $5 \% \mathrm{CO}_{2}$. For the siRNA silencing, cells were treated with the following previously described siRNA sequences (13) targeting the human ACY1 mRNA sequence: Sense, 5'-UCAACACGG UCACCACAUAGCCAGG-3' and antisense, 5'-CCUGGC UAUGUGGUGACCGUGUUGA-3'. The siRNA-NC was used as the negative control [scrambled control siRNA (cat. no. sc-37007) was obtained from Santa Cruz Biotechnology, Inc., Dallas, TX, USA]. Related recombined adenoviral expression vector were constructed by Invitrogen; Thermo Fisher Scientific, Inc. Lipofectamine RNAiMAX Transfection Reagent was used for the transfection of the siRNA (Invitrogen; Thermo Fisher Scientific, Inc.), according to the manufacturer's protocol. Briefly, $0.25 \times 10^{6}$ cells per well (6-well plate) were seeded prior to transfection. The transfection reagent was diluted by the medium according to the kit protocol and then the siRNA was added to the diluted transfection reagent at a 1:1 ratio. The cocktail was subsequently incubated for $5 \mathrm{~min}$ at room temperature and added to the cells. The transfection

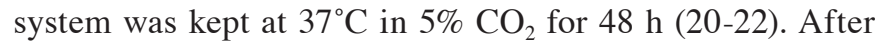
$48 \mathrm{~h}$, downregulation of ACY1 was measured by qPCR Western blotting was also performed to test the target protein in order to ensure knockdown efficiency.

Western blot analysis. Western blotting was performed to study the protein expression levels of ACY1, and its downstream
Table I. Clinicopathological characteristics of the patients.

\begin{tabular}{lcc}
\hline Characteristic & $\begin{array}{c}\text { Patients with } \\
\text { colorectal cancer }\end{array}$ & $\begin{array}{c}\text { Healthy } \\
\text { control group }\end{array}$ \\
\hline $\begin{array}{l}\text { Age (mean } \pm \text { SD; } \\
\text { range, years) }\end{array}$ & $52.3 \pm 12.1 ; 32-69$ & $50.1 \pm 13.8 ; 30-68$ \\
$\begin{array}{l}\text { Gender (no. } \\
\text { of patients) }\end{array}$ & & \\
Male & 81 & 72 \\
Female & 51 & 48 \\
TNM stage (no. & & \\
of patients) & & \\
Stage I & 44 & N/A \\
Stage II & 62 & N/A \\
Stage III & 18 & N/A \\
Stage IV & 8 & N/A \\
\hline
\end{tabular}

TNM, tumor-node-metastasis; N/A, not applicable.

and regulatory proteins, in the clinical samples and in cells in vitro. A total of $1 \mathrm{mg}$ tissue was solubilized in $100 \mu \mathrm{l}$ lysis buffer (30 mM Tris, $2 \mathrm{M}$ Thiourea, 4\% CHAPS, $7 \mathrm{M}$ urea; $\mathrm{pH} \mathrm{8.5)} \mathrm{on} \mathrm{ice} \mathrm{for} 30 \mathrm{~min}$. The lysis buffer was subsequently centrifuged at 1,000 x $g$ for $15 \mathrm{~min}$ and the supernatant was collected for further testing. Subsequently, western blotting was performed as previously described (18), and primary antibodies were used for incubation at $4^{\circ} \mathrm{C}$ overnight and secondary antibodies at room temperature for $1 \mathrm{~h}$. Primary antibodies targeting the following proteins were used: ACY1 (1:1,000; mouse; monoclonal; cat. no. ab54960; Abcam, Cambridge, UK); Transforming growth factor- $\beta 1$ (TGF- $\beta 1 ; 1: 1,000$; cat. no. 3709s; rabbit; monoclonal; Cell Signaling Technology, Inc., Danvers, MA, USA); extracellular signal-related kinase 1 (ERK1; 1:1,000; cat. no. 4094; rabbit; monoclonal; Cell Signaling Technology, Inc.); phosphorylated-ERK1 (p-ERK1; 1:1,000; cat. no. 4370; rabbit; monoclonal; Cell Signaling Technology, Inc.); and $\beta$-actin (1:1,000; cat. no. 8H10D10; mouse; monoclonal; Cell Signaling Technology, Inc.). The following secondary antibodies were used: anti-rabbit IgG-biotin (cat. no. BA1020); and anti-mouse IgG-biotin (cat. no. BM2001) (both Boster Biological Technology, Wuhan, China). Bands were detected using the DAB Chromogenic Reagent kit (cat. no. AR1021; Boster Biological Technology).

Serum ACY1 concentration ELISA. Serum levels of ACY1 protein were measured by ELISA. Prior to the ELISA, serum samples were centrifuged at $800 \times g$ at $4^{\circ} \mathrm{C}$ for $10 \mathrm{~min}$ and the supernatant was collected. The ELISA was then conducted using the ACY1 ELISA kit (cat. no. ABIN417990; Cloud-Clone Corporation, Houston, TX, USA), according to the manufacturer's protocol.

Cell Counting Kit-8 (CCK-8) and in vitro scratch assays. The effect of ACY1 knockdown by siRNA on apoptosis and proliferation in HT-29 cells in vitro was determined by the CCK- 8 and scratch assays, respectively. For the CCK- 8 assay, the 
A

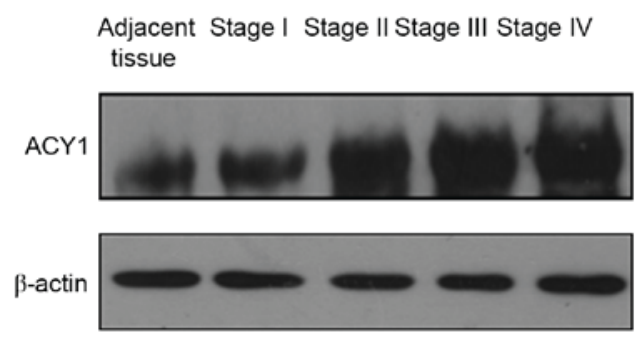

$\mathrm{C}$

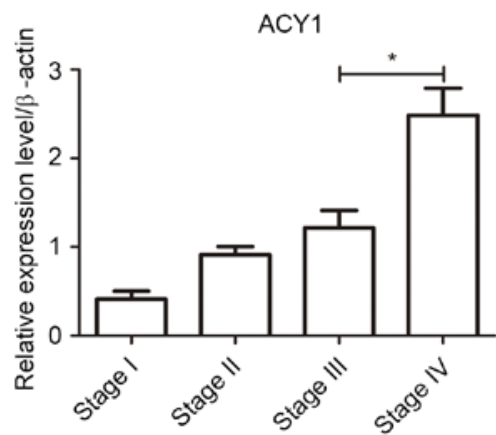

B

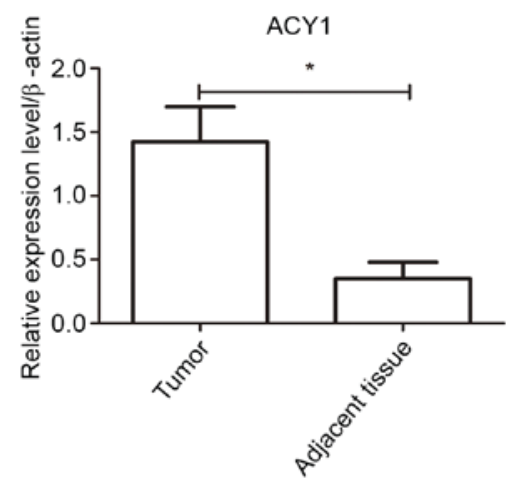

$\mathrm{D}$

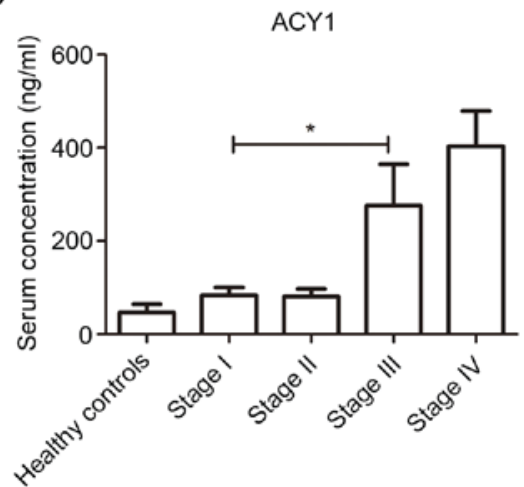

Figure 1. Overexpression of ACY1 in colorectal cancer. (A) Western blot analysis of ACY1 protein expression in clinical specimens from patients with tumors of different TNM stages. (B) ACY1 mRNA expression in patients with colorectal cancer. (C) ACY1 mRNA expression in clinical specimens from patients with tumors of different TNM stages. (D) Serum concentration of ACY1 protein in patients with tumors of different TNM stages. "P<0.05. ACY1, aminoacylase 1; TNM, tumor-node-metastasis.

CCK-8 kit (cat. no. CK04-11; Dojindo, Rockville, Maryland) was used. A total of 5,000 cells per well were seeded into a 96-well plate and incubated for $24 \mathrm{~h}$ prior to the test. The test was then performed and cell density measured according to the manufacturer's protocol. For the scratch assay, $1 \times 10^{6}$ cells were seeded into $60-\mathrm{mm}$ cell culture dishes. Following $6 \mathrm{~h}$ of culturing, as previously described, cell monolayers were scraped in a straight line with a sterilized p200 pipet tip and immediately washed with $1 \mathrm{ml}$ McCoy's 5A medium (Gibco; Thermo Fisher Scientific, Inc.; cat. no. 16600-082). Cell monolayers were observed and measured every $12 \mathrm{~h}$ for a total of $48 \mathrm{~h}$. The width of the scratch was recorded at 5 different points at each time point and the cell migration ability was calculated as the width of the scratch at each time point, as a percentage of the width at $0 \mathrm{~h}$.

Statistical analysis. Data was analyzed using SPSS software v17.0 (SPSS, Inc., Chicago, IL, USA). The association between ACY1 mRNA expression and the tumor-node-metastasis (TNM) stage of tumors was assessed using the $\chi^{2}$ test. Differences in mRNA expression between groups were compared using the Student's t-test and post hoc test (Scheffe). $\mathrm{P}<0.05$ was considered to indicate a statistically significant difference.

\section{Results}

ACY1 expression is increased in tumor specimens and serum samples from patients with colorectal cancer. Western blotting (Fig. 1A), RT-qPCR (Fig. 1B and C) and ELISA (Fig. 1D) were used to measure the mRNA and protein expression levels of ACY1 from tumor specimens and serum samples of patients with colorectal cancer. This demonstrated that, compared with adjacent normal tissues, ACY1 protein expression was markedly increased in the tumor tissues (Fig. 1A). RT-qPCR analysis revealed a similar increase in ACY1 mRNA expression, with relative ACY1 mRNA expression significantly increased in tumor tissue compared with normal adjacent tissue $(\mathrm{P}=0.023$; Fig. 1B). Furthermore, a notable increase in the serum concentration of ACY1 protein was observed in patients with colorectal compared with the healthy controls (Fig. 1D).

Association between ACY1 expression and TNM stage in colorectal cancer. Out of the 132 patients in the cohort of this study, 44 patients had TNM stage I tumors, 62 had stage II tumors, 18 had stage III tumors and 8 had stage IV tumors. The levels of ACY1 mRNA and protein expression were compared between these groups. This revealed that tissue specimens (Fig. 1C) and serum samples (Fig. 1D) from patients with TNM stage II and IV tumors had markedly increased ACY1 expression compared with those with stage I and II disease. In addition, ACY1 mRNA expression was significantly increased in stage IV compared with stage II tumor specimens $(\mathrm{P}=0.037$; Fig. 1C). Furthermore, the serum concentration of ACY1 protein was significantly increased in in patients with stage III tumors compared with those with stage I tumors and healthy controls ( $\mathrm{P}=0.025$; Fig. 1D).

Inhibiting ACY1 expression reduces colorectal cancer cell proliferation and increases apoptosis rates in vitro. To 
A

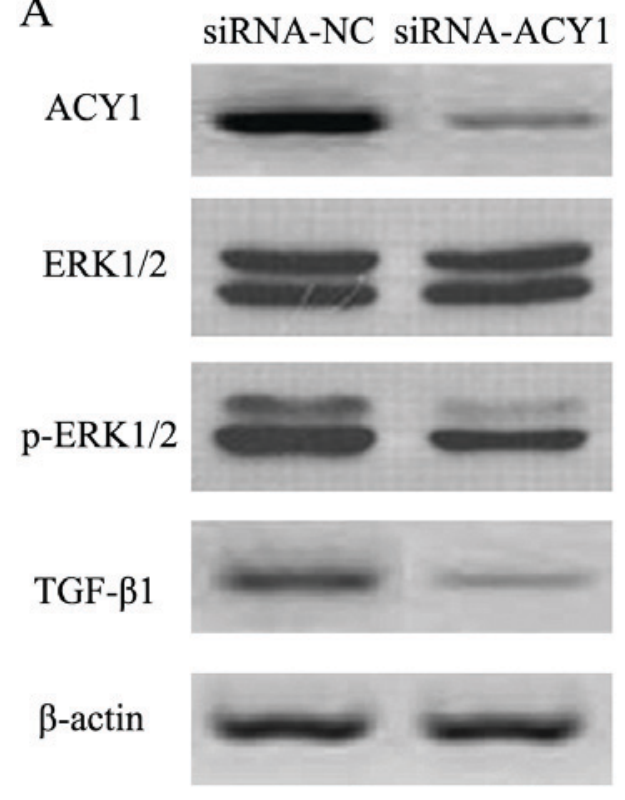

$\mathrm{B}$
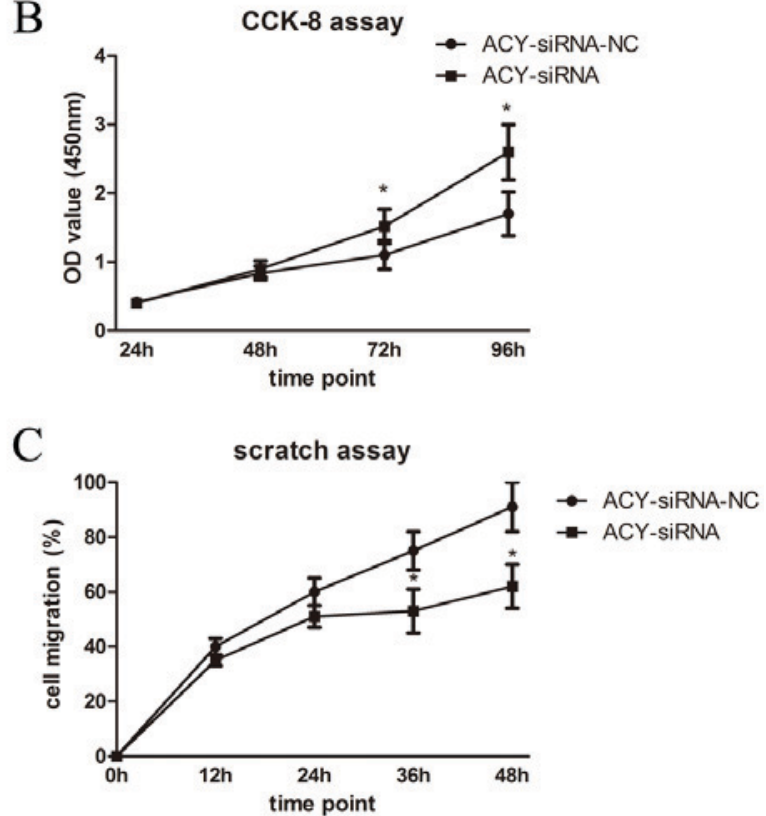

Figure 2. Protein expression and growth of human colorectal cancer HT-29 cells following siRNA silencing of ACY1. (A) Western blot of protein expression level of ACY1 and its potential downstream proteins. (B) CCL-8 assay for apoptosis. P $<0.05$ vs. siRNA-NC at 72 and $96 \mathrm{~h}$. (C) Scratch assay for cell proliferation. P<0.05 vs. siRNA-NC at 36 and 48 h. ACY1, aminoacylase 1; siRNA, small interfering RNA; ERK1/2, X; p-ERK1/2, X; TGF- $\beta 1$, transforming growth factor- $\beta 1$; CCK-8, Cell Counting Kit-8; NC, negative control.

investigate the role of ACY1 in colorectal cancer, siRNA silencing was performed. As shown in Fig. 2A, siRNA silencing markedly reduced $\mathrm{ACY} 1$ protein expression in the HT-29 cells. The CCK- 8 and scratch assays revealed that following knockdown of ACY1 expression, the cancer cells exhibited increased rates of apoptosis (Fig. 2B; $96 \mathrm{~h}$ time point siRNA-NC $1.70 \pm 0.32$ vs. siRNA-ACY1 $2.60 \pm 0.40 ; \mathrm{P}=0.036$ ) and reduced proliferation (Fig. $2 \mathrm{C} ; 48 \mathrm{~h}$ time point siRNA-NC $91 \pm 9.0$ vs. siRNA-ACY1 $62 \pm 8.0 ; \mathrm{P}=0.003)$.

Knockdown of ACY1 increases expression of ERK and TGF- $\beta 1$ in HT-29 cells. In order to study the effects of ACY1 knockdown, the protein expression levels of ERK1, p-ERK1 and TGF- $\beta 1$ were measured in cells treated with ACY-targeting siRNA. Notably, the protein expression levels of the well-known protein TGF- $\beta 1$ along with the ERK $1 / 2$ phosphorylation, that are known to be upregulated in colorectal cancer $(23,24)$, were observed to decrease following knockdown of ACY1 expression (Fig. 2A). This indicates that p-ERK1 and TGF- $\beta 1$ may function downstream of ACY1 to affect tumorigenesis.

\section{Discussion}

The present study identified a significantly increased expression of ACY1 mRNA in tumor tissue specimens and serum samples from patients with colorectal cancer. In addition, a similar increase was observed in ACY1 protein levels. Although no previous studies, to the best of our knowledge, have suggested that there is an association between ACY1 expression and the severity of colorectal cancer, the results of the current study demonstrated that the level of ACY1 mRNA and protein is positively associated with the TNM stage of the tumor. Previously, ACY1 has been widely recognized for its catalytic activity in the acylation of $\mathrm{N}$-terminal amino acids $(6-8,20-22)$. ACY1 is widely expressed in different organs and previous studies have shown that in the human digestive tract ACY1 is particularly abundant in epithelial cells, but not in stromal cells $(23,24)$.

Although the role of ACY1 in aminoacylation is well known, its role in cancer remains unclear. A previous study in SCLC cell lines suggested that expression of ACY1 would be reduced to an undetectable level in SCLC (13) and similar results were observed in liver cancer (17). These results indicated that ACY1 expression would be reduced throughout the process of tumor progress. However, the results of the present study on colorectal cancer found the opposite. A significant increase in ACY1 mRNA levels was observed in colorectal cancer specimens compared with healthy adjacent tissue, and a similar effect was observed for ACY1 tumor and serum protein levels. In order to study the effects of this increase, siRNA was used to knockdown ACY1 expression in a colorectal cancer cell line. The results of this knockdown indicated that inhibition of ACY1 expression leads to a reduction in proliferation and increase in apoptosis in colorectal cancer cells. These results indicate that in colorectal cancer ACY1 has a tumor-promoting function.

Previous reports have shown a novel proliferation-promoting function of ACY1 in numerous cell lines, including NIH/3T3, HEK293 and COS-7 cells $(23,25)$. However, little is known about the mechanisms underlying this function. It has been noted that Sphingosine kinase type 1 (SphK1), an anti-apoptosis protein can bind to ACY1 and enhance its anti-apoptotic effect $(23,26)$. However, this alone is not sufficient evidence to demonstrate the function of ACY1 in cancer. The current study focused the association between ACY1 expression and the expression of ERK1, which is a key component of an important signaling pathway involved in cell growth and immune responses, respectively (27-29). Previous studies 
have confirmed that SphK1 functions through this pathway and results in increased cell proliferation (28,30-32), which indicates that ERK functions downstream of SphK1. Notably, a microarray study revealed that ACY1 silencing could induce a significant increase in ERK expression (33). These results all indicate that ACY1 functions via the ERK pathway. Furthermore, activation of ERK increases the expression of TGF- $\beta$, another important factor involved in tumor progress $(13,15-17)$, and TGF- $\beta$ could therefore directly attenuate the immune system and promote tumor proliferation $(13,17)$. Thus, decreased expression of TGF- $\beta$ could lead to tumor inhibition. In the present study, similar results were identified in colorectal cancer cells following ACY1 knockdown, which resulted in decreased ERK activation and decreased TGF- $\beta$ expression, inhibiting tumorigenesis.

The present study measured the expression of ERK1 in clinical specimens and in cells in vitro. As predicted, this revealed that silencing of ACY1 reduced the level of ERK1 expression in colorectal cancer tissue, which indicates that there is an overexpression of ERK1 along with ACY1 in colorectal cancer. In addition, since it is well known that activation of ERK leads to increased TGF- $\beta 1$ expression $(13,15,16)$, the association between ACY1 and TGF- $\beta 1$ expression was measured, which revealed a positive association between the two. The results of the present study indicate that the ERK and TGF- $\beta 1$ function downstream of ACY1 and facilitate the tumor-promoting effect of ACY1.

The early diagnosis and prognosis of colorectal cancer is an area of intense study worldwide. The most frequently used strategy for diagnosing colorectal cancer is the measurement of serum concentrations of diagnostic biomarkers, including carcinoembryonic antigen and CA-125 (33). In the current study, serum levels of ACY1 protein were upregulated in patients with colorectal cancer. The results showed a positive correlation between serum ACY1 concentration and colorectal cancer progression, which may be due to increased expression in the tumors. Notably, a study on renal transplantation suggested that serum ACY1 levels could be used as a biomarker for measuring the severity of inflammation following surgery (21). As tumors and transplantation can trigger immune responses, changes in the level of serum ACY1 may reflect immune reactions occurring within the body. Therefore, the findings of the current study suggest that ACY1 could be used as a diagnostic biomarker of colorectal cancer.

In conclusion, the results of the present study identified that expression of ACY1 is significantly increased in colorectal cancer and is positively associated with tumor progression. In addition, the results indicate that ACY1 promotes tumor cell proliferation through activation of the ERK pathway, which results in increased expression of TGF- $\beta 1$, leading to the promotion of tumor growth and inhibition of immune responses. These results suggest that serum ACY1 concentration could be used as a novel biomarker for the diagnosis of colorectal cancer.

\section{References}

1. Ferlay J, Shin HR, Bray F, Forman D, Mathers C and Parkin DM: Estimates of worldwide burden of cancer in 2008: GLOBOCAN 2008. Int J Cancer 127: 2893-2917, 2010.

2. American Cancer Society. Cancer facts \& figures 2010. American Cancer Society, Atlanta, GA, 2010.
3. Liao X, Lochhead P, Nishihara R, Morikawa T, Kuchiba A, Yamauchi M, Imamura Y, Qian ZR, Baba Y, Shima K, et al: Aspirin use, tumor PIK3CA mutation, and colorectal-cancer survival. N Engl J Med 367: 1596-1606, 2012.

4. Siegel R, Desantis C and Jemal A: Colorectal cancer statistics, 2014. CA Cancer J Clin 64: 104-117, 2014.

5. Van Cutsem E, Köhne CH, Láng I, Folprecht G, Nowacki MP, Cascinu S, Shchepotin I, Maurel J, Cunningham D, Tejpar S, et al: Cetuximab plus irinotecan, fluorouracil, and leucovorin as first-line treatment for metastatic colorectal cancer: Updated analysis of overall survival according to tumor KRAS and BRAF mutation status. J Clin Oncol 29: 2011-2019, 2011.

6. Sass JO, Mohr V, Olbrich H, Engelke U, Horvath J, Fliegauf M, Loges NT, Schweitzer-Krantz S, Moebus R, Weiler P, et al: Mutations in ACY1, the gene encoding aminoacylase 1, cause a novel inborn error of metabolism. Am J Hum Genet 78: 401-409, 2006.

7. Maceyka M, Nava VE, Milstien S and Spiegel S: Aminoacylase 1 is a sphingosine kinase 1-interacting protein. FEBS Lett 568: 30-34, 2004.

8. Naylor SL, Elliott RW, Brown JA and Shows TB: Mapping of aminoacylase-1 and beta-galactosidase-A to homologous regions of human chromosome 3 and mouse chromosome 9 suggests location of additional genes. Am J Hum Genet 34: 235-244, 1982.

9. Lindner HA, Lunin VV, Alary A, Hecker R, Cygler M and Ménard R: Essential roles of zinc ligation and enzyme dimerization for catalysis in the aminoacylase-1/M20 family. J Biol Chem 278: 44496-44504, 2003.

10. Miller YE, Minna JD and Gazdar AF: Lack of expression of aminoacylase-1 in small cell lung cancer. Evidence for inactivation of genes encoded by chromosome 3p. J Clin Invest 83: 2120-2124, 1989.

11. Zhong Y, Onuki J, Yamasaki T, Ogawa O, Akatsuka S and Toyokuni S: Genome-wide analysis identifies a tumor suppressor role for aminoacylase 1 in iron-induced rat renal cell carcinoma. Carcinogenesis 30: 158-164, 2009.

12. Park KS, Cho SY, Kim H and Paik YK: Proteomic alterations of the variants of human aldehyde dehydrogenase isozymes correlate with hepatocellular carcinoma. Int J Cancer 97: 261-265, 2002.

13. Wei X, Li J, Xie H, Ling Q, Wang J, Lu D, Zhou L, Xu X and Zheng S: Proteomics-based identification of the tumor suppressor role of aminoacylase 1 in hepatocellular carcinoma. Cancer Lett 351: 117-125, 2014.

14. Wang Z, Hu P, Tang F and Xie C: HDAC6-mediated EGFR stabilization and activation restrict cell response to sorafenib in non-small cell lung cancer cells. Med Oncol 33: 50, 2016.

15. Yijing L, Liu H, Yuan C, Wang B, Ren M, Yan L, Wang X and Zhang J: The effects of qindan-capsule-containing serum on the TGF- $\beta 1 /$ ERK signaling pathway, matrix metalloproteinase synthesis and cell function in adventitial fibroblasts. Pharm Biol 51: 712-721, 2013.

16. Zhou Q, Zeng R, Xu C, Liu L, Chen L, Kou P, Pei G, Bai S, Zhang Y, Li C, et al: Erbin inhibits TGF- $\beta 1$-induced EMT in renal tubular epithelial cells through an ERK-dependent pathway. J Mol Med (Berl) 90: 563-574, 2012.

17. Shirasawa $S$, Yoshie $S$, Yue $F$, Ichikawa $H$, Yokoyama $T$, Nagai M, Tomotsune D, Hirayama M and Sasaki K: Pancreatic exocrine enzyme-producing cell differentiation via embryoid bodies from human embryonic stem cells. Biochem Biophys Res Commun 410: 608-613, 2011.

18. Valente KN, Choe LH, Lenhoff AM and Lee KH: Optimization of protein sample preparation for two-dimensional electrophoresis. Electrophoresis 33: 1947-1957, 2012.

19. Marchbank T, Ojobo E, Playford CJ and Playford RJ: Reparative properties of the traditional Chinese medicine Cordyceps sinensis (Chinese caterpillar mushroom) using HT29 cell culture and rat gastric damage models of injury. Br J Nutr 105: 1303-1310, 2011.

20. Ferri L, Funghini S, Fioravanti A, Biondi EG, la Marca G, Guerrini R, Donati MA and Morrone A: Aminoacylase I deficiency due to ACY1 mRNA exon skipping. Clin Genet 86: 367-372, 2014.

21. Welberry Smith MP, Zougman A, Cairns DA, Wilson M, Wind T, Wood SL, Thompson D, Messenger MP, Mooney A, Selby PJ, et al: Serum aminoacylase-1 is a novel biomarker with potential prognostic utility for long-term outcome in patients with delayed graft function following renal transplantation. Kidney Int 84: 1214-1225, 2013.

22. Ali S and Sheerin NS: Biomarkers of acute injury: Predicting the long-term outcome after transplantation. Kidney Int 84: 1072-1074, 2013. 
23. Shi H, Hayes MT, Kirana C, Miller RJ, Keating JP and Stubbs RS Overexpression of aminoacylase 1 is associated with colorectal cancer progression. Hum Pathol 44: 1089-1097, 2013.

24. Pérez-Pérez R, García-Santos E, Ortega-Delgado FJ, López JA, Camafeita E, Ricart W, Fernández-Real JM and Peral B: Attenuated metabolism is a hallmark of obesity as revealed by comparative proteomic analysis of human omental adipose tissue. J Proteomics 75: 783-795, 2012.

25. Weissenberger S, Schultheis C, Liewald JF, Erbguth K, Nagel G and Gottschalk A: $P A C \alpha$-an optogenetic tool for in vivo manipulation of cellular cAMP levels, neurotransmitter release, and behavior in Caenorhabditis elegans. J Neurochem 116: 616-625, 2011.

26. Sommer A, Christensen E, Schwenger S, Seul R, Haas D, Olbrich H, Omran H and Sass JO: The molecular basis of aminoacylase 1 deficiency. Biochim Biophys Acta 1812: 685-690, 2011

27. De Luca A, Maiello MR, D'Alessio A, Pergameno $M$ and Normanno N: The RAS/RAF/MEK/ERK and the PI3K/AKT signalling pathways: Role in cancer pathogenesis and implications for therapeutic approaches. Expert Opin Ther Targets 16 (Suppl 2): S17-S27, 2012.

28. Wang VE, McCormick F and Settleman J. Hyperactive AKT pathway and reactivation of the MAPK/ERK pathway in melanoma cells resistant to dual BRAF and MEK inhibition. Cancer Res 74 (Suppl): S3698, 2014.
29. Santarpia L, Lippman SM and El-Naggar AK: Targeting the MAPK-RAS-RAF signaling pathway in cancer therapy. Expert Opin Ther Targets 16: 103-119, 2012.

30. Deng W, Sui H, Wang Q, He N, Duan C, Han L, Li Q, Lu M and Lv S: A Chinese herbal formula, Yi-Qi-Fu-Sheng, inhibits migration/invasion of colorectal cancer by down-regulating MMP-2/9 via inhibiting the activation of ERK/MAPK signaling pathways. BMC Complement Altern Med 13: 65 , 2013.

31. Prokudin I, Stasyk T, Rainer J, Bonn GK, Kofler R and Huber LA: Comprehensive proteomic and transcriptomic characterization of hepatic expression signatures affected in p14 liver conditional knockout mice. Proteomics 11: 469-480, 2011.

32. Alshaker H, Sauer L, Monteil D, Ottaviani S, Srivats S, Böhler T and Pchejetski D: Therapeutic potential of targeting SK1 in human cancers. Adv Cancer Res 117: 143-200, 2013.

33. Jain P, Mondal SK, Sinha SK, Mukhopadhyay $M$ and Chakraborty I: Diagnostic and prognostic significance of different mucin expression, preoperative CEA, and CA-125 in colorectal carcinoma: A clinicopathological study. J Nat Sci Biol Med 5: 404-408, 2014. 